\title{
Diseñar un manual de procedimientos de créditos y cobranzas para reducir la morosidad en la empresa darcell servicios integrados S.R.L. en la ciudad de Jaén en el periodo 2020
}

\author{
Br. Paquita Mariela Bernal Neyra. \\ pbernalne@ucvvirtual.edu.pe \\ https://orcid.org/0000-0003-0679-7471
}

Br. Lenin Smith Segura Ojeda.

lseguraoj@ucvvirtual.edu.pe https://orcid.org/0000-0001-9386-7815

Mg. Roberto Carlos Oblitas Otero. https://orcid.org/0000-0002-6541-7005 roblitas@ucvvirtual.edu.pe

Universidad César Vallejo

Moyobamba - Perú

\section{RESUMEN}

El presente trabajo de investigación se elaboró a la empresa denominada Darcell Servicios Integrados S.R.L. que se encuentra ubicada en la calle San Martín N 1811 , en la localidad de Jaén, Cajamarca. Esta empresa se dedica a la realización de servicios: microcréditos y créditos por convenio con instituciones del sector público, servicios profesionales de contabilidad y administración, y otras actividades conexas. Esta tesis tiene como objetivo principal analizar si diseñar un manual de procedimientos de créditos y cobranzas va permitir reducir la morosidad en la empresa Darcell Servicios Integrados S.R.L., en la ciudad de Jaén, en el periodo 2020; y se elaboró mediante el método cuantitativo, tipo de investigación aplicada, de alcance descriptivo, diseño no experimental; y se elaboró mediante el método cuantitativo, tipo de investigación aplicada, de alcance descriptivo, diseño no experimental. El motivo por el cual se diseñó el manual de procedimientos de créditos y cobranzas, porque si bien es cierto la empresa tiene formas y criterios de evaluación de créditos, estas no se encontraban plasmadas en un manual de procedimientos de créditos y aún más la forma de cobranza de créditos vencidos no estaba funcionando a la perfección ya que el índice de morosidad y atraso en la cuota crediticia 
se incrementa. En conclusión, se obtuvo que un manual de procedimientos de créditos y cobranzas es de mucha importancia para la empresa ya que permitió un buen proceso de evaluación al cliente, dando como resultado disminución de créditos vencidos y por ende la disminución gradual de morosidad.

Palabras clave: manual de créditos y cobranzas; índice de morosidad; procedimientos de créditos y cobranzas; reducir. 


\title{
Design a manual of credit and collection procedures to reduce delay in the company Darcell Servicios Integrados S.R.L. In the city of Jaén in the period 2020
}

\begin{abstract}
This research work was prepared at the company called Darcell Servicios Integrados S.R.L. which is located at Calle San Martín No. 1811, in the town of Jaén, Cajamarca. This company is dedicated to providing services: microcredits and loans by agreement with public sector institutions, professional accounting and administration services, and other related activities. The main objective of this thesis is to analyze whether designing a loan and collection procedures manual will reduce delinquency in the company Darcell Servicios Integrados S.R.L., in the city of Jaén, in the period 2020; and it was elaborated by means of the quantitative method, type of applied investigation, of descriptive scope, non-experimental design; and it was developed using the quantitative method, type of applied research, descriptive scope, non-experimental design. The reason why the credit and collections procedures manual was designed, because although the company has forms and criteria for evaluating credits, these were not reflected in a credit procedures manual and even more so the form of Collection of overdue loans was not working perfectly as the delinquency and delinquency rate in the loan installment increased. In conclusion, it was obtained that a manual of credit and collection procedures is of great importance for the company since it allowed a good evaluation process to the client, resulting in a decrease in overdue loans and therefore a gradual decrease in delinquency.
\end{abstract}

Keywords: manual of credits and collections; delinquency rate; credit and collection procedures; reduce.

Artículo recibido: 05 octubre. 2021 Aceptado para publicación: 02 noviembre 2021 Correspondencia: bernalneyra.18@gmail.com Conflictos de Interés: Ninguna que declarar 


\section{INTRODUCCIÓN}

En el entorno Internacional, en el continente Europeo con el fin de minimizar los préstamos morosos se realiza apresuradamente la limpieza de los balances bancarios y se pronostica que continúen, según el primer informe de la comisión Europea. En los últimos años, pese a que el banco de España estableció estrictamente las normas contables para aquellos préstamos en condición de morosidad, la cartera de clientes morosos en préstamos por consumo ha incrementado considerablemente. Considerando como objeto general la verificación de balances de los bancos y las políticas económicas por parte del Banco Central Europeo, se concluyó la contribución de la reducción de las tasas de morosidad y la permanencia en el tiempo, produce que la crisis económica y financiera vaya disminuyendo con el tiempo. Debido al control y la imposición de normas de los bancos nacionales y del ente regulador europeo está produciendo resultados positivos, así como la prevista subida de tipos por parte del Banco Central Europeo que aumentarán los márgenes en los balances de los bancos, nos augura unas buenas perspectivas del sector bancario, que únicamente ha de pasar la prueba de la reducción de estímulos por parte del Banco Central Europeo. (Moreno, 2018).

En EE.UU el crédito se puede controlar a través de la otorgación limitada del monto desembolsado al cliente, esto ocasiona un mayor control en el consumo y la inversión. Es decir, si el deterioro del escenario económico se da junto con la reducción del precio de los activos, por ende, disminuye el valor de las garantías en las que los prestatarios respaldan su crédito, de tal manera, reducirá la capacidad de endeudamiento. Por otro lado, en los tramos finales de las expansiones económicas se suelen endurecer las condiciones de crédito, dado que se anticipa un deterioro de las expectativas de crecimiento y, por lo tanto, una mayor probabilidad de impago. (Murillo Gili, 2019).

En el entorno nacional, en el Perú es un caso distinto a los demás países de América Latina. Para IPSOS mediante las investigaciones y datos recolectados, actualmente el 41 $\%$ de la población Perú urbano, adultos entre la edad de 18 a 70 años cuenta con créditos en las distintas entidades financieras. En el año 2014, según los datos brindados por el Banco Mundial, de cada 10 peruanos solo 2 tienen una cuenta bancaria. Para Asbanc, en su informe brindado al finalizar el año 2017 determinó que el $35.93 \%$ de la población peruana conforma el sistema financiero. Es decir, incremento de cada 10 peruanos, 4 hacían uso de un producto bancario. Ya en octubre del 2018 la morosidad en los créditos 
de consumo y créditos hipotecarios incrementaron. Inculcar la cultura financiera a la población crediticia permitirá reducir el índice de morosidad en las entidades financieras. Asimismo, dando a conocer al 59\% de población de las zonas urbanas la importancia de la bancarización de sus recursos monetarios. (Bobadilla, 2019).

A nivel local la empresa Darcell Servicios Integrados S.R.L, en la actualidad viene sufriendo con un elevado índice de morosidad, esto se viene dando desde los últimos cuatro años anteriores, estos problemas se vienen produciendo debido a que no cuenta con un manual adecuado de procedimientos de créditos y cobranzas, lo cual genera que gerencia sea el que autorice la aprobación y colocación de créditos según su criterio y muchas veces se extralimita en los montos aprobados y en las cantidad de cuotas fijadas. Dando como consecuencia créditos vencidos sin cancelar, y capital no recuperado y por ende reflejo de pérdida de utilidades en la empresa. De allí tiene como principio el desarrollo del presente trabajo de investigación lo cual nos permitirá dar alternativas eficientes de créditos y cobranzas, teniendo en cuenta todo lo expuesto.

\section{Problema general}

- Teniendo en cuenta lo explicado se determinó el siguiente problema principal: ¿Qué efectos produce diseñar un manual de procedimientos de créditos y cobranzas en el proceso de reducción de la morosidad en la empresa Darcell Servicios Integrados S.R.L., en la ciudad de Jaén, en el periodo 2020 ?

\section{Problemas específicos}

- ¿Cómo optimizar los criterios de evaluación crediticia en el manual de procedimientos de créditos y cobranzas para reducir la morosidad en la empresa Darcell Servicios Integrados S.R.L.?

- ¿Cómo obtener la eficiencia en la gestión de cartera de clientes, créditos y cobranzas con relación a la morosidad en la empresa Darcell Servicios Integrados S.R.L.?

- ¿Cómo implantar estrategias en el manual de procedimientos de créditos y cobranzas para disminuir la morosidad de la empresa Darcell Servicios Integrados S.R.L.?

\section{Justificación}

La siguiente investigación tiene como justificación: se orientó en diseñar un manual de créditos y cobranzas para la empresa "Darcell Servicios Integrados S.R.L.", cuya finalidad es mejorar los procesos del área de finanzas, además dicho manual se desarrolló aplicando criterios de evaluación para el otorgamiento de créditos, analizando que tan 
eficiente es la gestión de cartera de clientes, créditos y cobranzas, implantando estrategias de políticas de créditos y cobranzas, analizando las funciones de cada uno de los empleados existentes y que sus actividades cotidianas permitan disminuir la morosidad. Se dio con la finalidad de establecer criterios de evaluación y estrategias de políticas de créditos y cobranzas para disminuir el saldo deudor, de manera que permita que la realización de las actividades operativas se trate de forma eficiente y organizada.

\section{Objetivo general}

- Analizar si el diseño de un manual de procedimientos de créditos y cobranzas permite reducir la morosidad en la empresa Darcell Servicios Integrados S.R.L., en la ciudad de Jaén, en el periodo 2020.

\section{Objetivos específicos}

a) Establecer procesos y criterios de evaluación crediticia en el manual de procedimientos de créditos y cobranzas para reducir la morosidad en la empresa Darcell Servicios Integrados S.R.L.

b) Implementar la gestión de cartera de clientes, créditos y cobranzas con relación a la morosidad en la empresa Darcell Servicios Integrados S.R.L.

c) Aplicar estrategias en el manual de procedimientos de créditos y cobranzas para disminuir la morosidad de la empresa Darcell Servicios Integrados S.R.L.

\section{Hipótesis general}

- Diseñar el manual de procedimientos de créditos y cobranzas, permitió una mejor evaluación crediticia y se logró reducir un gran porcentaje la morosidad de deudores de la empresa Darcell Servicios Integrados S.R.L.

\section{Hipótesis específicas}

- Establecer procesos y criterios de evaluación crediticia en el manual de procedimientos de créditos y cobranzas, permitirá reducir la morosidad en la empresa Darcell Servicios Integrados S.R.L.

- Implementar la gestión de cartera de clientes, créditos y cobranzas, se obtendrá un mejor control de la morosidad en la empresa Darcell Servicios Integrados S.R.L.

- Aplicar estrategias en el manual de procedimientos de créditos y cobranzas, disminuirá la morosidad de la empresa Darcell Servicios Integrados S.R.L. 


\section{Antecedentes}

Solano, B. \& Gómez, G. (2016). Donde en su tesis "Propuesta de un manual de créditos y cobranzas para mejorar la liquidez" (Artículo científico) Guayaquil, Ecuador. Siendo un tipo de investigación básica- cuantitativa, además su objetivo general el diseño de un modelo estratégico de procesos dentro de un manual de créditos y cobranzas con a finalidad de mejorar la rentabilidad de la empresa, en el cual concluyó que la implementación del manual ocasiona la reducción de créditos morosos y reduce en un gran porcentaje disminuye la falta de pagos y/o créditos vencidos.

Por otra parte Vega Barrios, K. (2019). En su tesis "Company portfolio procedure manual colpromed Sas" (Artículo científico) Bucaramanga, Colombia. Siendo un tipo de investigación básica, tipo de estudio fue descriptivo, el cual tiene como objetivo principal la elaboración de un informe del estado actual de la cartera de Colpromed sas, para ello elaboró un manual de cartera que contenga procesos, políticas y planes de realización para con los créditos. El cual concluye que obtener un portafolio detallado correctamente con los datos importantes y relevantes de los clientes permite a la empresa un proceso de cobranza mucho más fácil y eficaz por ende contribuye con una buena recaudación de créditos morosos el cual disminuirá el riesgo de la cartera crediticia.

Paredes, E. (2019), “Calificación crediticia y otorgamiento de créditos en la Banca Comercial de Lima Metropolitana, 2011 - 2018”. (Artículo científico) Lima, Perú. El tipo de investigación fue de tipo correlacional; tiene como objetivo establecer la relación que hay entre calificación crediticia y otorgamiento de créditos en la Banca Comercial de Lima Metropolitana. De toda la investigación se concluyó que es de mucha importancia verificar el riesgo crediticio para las entidades financieras, de eso depende administrarlo, teniendo en cuenta que la tasa de interés activa del crédito, depende del monto dado.

Coronel Huamán, L. (2016), “Estrategias de cobranza y su relación con la morosidad en la entidad financiera Mi Banco- Jaén 2015” (Artículo científico) Jaén, Perú. Investigación cuantitativa, tiene como objetivo general diagnosticar la relación que existe entre las estrategias de cobranza en la reducción de la morosidad en la entidad financiera Mi Banco- Jaén; la cual llegó a la conclusión que en la entidad financiera Mibanco se debe llevar a cabo un análisis riguroso de la situación económica financiera de sus clientes, a la vez se debe proporcionar alternativas de solución para los diferentes tipos de deudores, con la finalidad de reestablecer la cultura de pago de sus clientes, por otra parte se obtuvo 
que el problema de morosidad en las entidades financieras es porque los clientes distribuyen mal su dinero, destinando a otros gastos y no al pago de sus préstamos; también se da por la falta de cultura financiera y por otros siniestros que suceden en el transcurso o vigencia del préstamo.

\section{Teorías}

- "Existe diversos estudios de investigación donde explican los factores que influyen en la rentabilidad de una entidad bancaria. El riesgo crediticio es uno de los factores determinantes que influye en gran magnitud, para ello contamos con el indicador principal en relación al total de préstamos y este viene hacer la morosidad”. Chavarín (2015).

- “Contar con un Manual de Procedimientos atribuye excelencia a la organización y/o área para la que se diseñó, también contribuye con los trabajadores permitiendo un correcto proceso en su trabajo logrando cumplir con los objetivos y misión de la empresa, contribuyendo con sus objetivos y logros personales". Vivanco (2017).

- "Toda empresa financiera que elabore y aplique un manual de procedimientos que contenga los procesos a seguir en el área de créditos y cobranzas permite el buen funcionamiento, mejora la forma de evaluación, contribuye con estrategias de cobranzas fortaleciendo las políticas y reglamentos de la empresa, dando como resultado una cartera de clientes óptima”. Strap \& Slao (2018).

La importancia del manual de procedimientos de mréditos y cobranzas es disponer procesos, normatividad, políticas que faciliten una buena evaluación crediticia, y por ende una obtención de cartera de clientes fieles y con buen historial de pago esto permitirá el buen funcionamiento y mejorará los procedimientos en el área de crédito y cobranzas, adicional a ello es importante la capacitación constante a los trabajadores de esta área. Strap \& Slao (2018).

\section{ESTRATEGIAS METODOLÓGICAS O MATERIALES Y MÉTODOS}

\section{Tipo de investigación}

El tipo de investigación de este trabajo es aplicada ya que se basa en buscar nuevos conocimientos, que permitan dar soluciones. Para Álvarez (2020), “una investigación aplicada es aquella investigación orientada a lograr un nuevo conocimiento destinado a permitir soluciones de problemas prácticos". 
Se utilizó el enfoque cuantitativo ya que "mediante el instrumento de recolección de datos, se analiza la cantidad de datos obtenidos para probar si la hipótesis formulada previamente es verídica, es un enfoque que permite hallazgos precisos”. Gallardo (2017).

\section{Diseño de investigación}

El siguiente informe de investigación se encuentra apoyado en el diseño de estudio no experimental. Según Mata (2019), “el investigador no manipula las variables, sino más bien se analiza causa- efecto sin incidir sobre la causa; es decir, se observa el fenómeno tal y como se presentan e influyen entre dichas". El objetivo es observar y describir cada una de las variables, el cual nos permitió plantear el diseño de un manual de procesos para ser aplicado en la empresa.

La investigación fue de nivel descriptivo, porque "se busca analizar los datos recolectados a través de la encuesta, para dar solución al problema". Ramos (2020).

\section{Población}

La población se encuentra conformada por todos los trabajadores (3 trabajadores) de la empresa Darcell Servicios Integrados S.R.L, de la ciudad de Jaén.

\section{Muestra}

La muestra está comprendida por el área de microcréditos; es decir, los 3 trabajadores. Se consideró el total de las personas que laboran debido a que todas se dedican a ejercer las mismas actividades.

\section{Muestreo}

El muestreo empleado fue el no probabilístico, pues permitió seleccionar casos peculiares de la población delimitando la muestra solo a este caso, además la muestra tomada fue muy pequeña.

\section{Técnicas e instrumentos de recolección de datos}

Las técnicas que se utilizó para el desarrollo del siguiente informe de tesis es la encuesta y se utilizó con la finalidad de recolectar los datos necesarios de los trabajadores de la empresa Darcell Servicios Integrados SRL.

La técnica de encuesta es la más utilizada para procesar la información que se requiere obtener en una investigación, ya que contribuye en la recopilación y elaboración de datos de modo rápido y eficaz.

\section{Procedimientos}

En el siguiente informe de investigación en la técnica de encuesta se elaboró un 
cuestionario con trece preguntas cerradas que es donde el encuestado se limita a elegir una respuesta definidas previamente en el cuestionario; las respuestas están totalmente precodificadas, las cuales se aplicó a los trabajadores del área de microcréditos, con la finalidad de conocer sus opiniones, sugerencias sobre el tema y definir resultados sobre la investigación.

\section{Método de análisis de datos}

Para analizar los datos estadísticos de la técnica ejecutada se ha utilizado el software Microsoft Excel donde se realizó una tabla para tabular los datos recolectados y gráfico para mostrar la cantidad representativa obtenida.

Para la presentación de la información recolectada se empleó las siguientes formas:

- Presentación escrita: Esta forma de presentación resalta la información principal y precisa para la determinación del tema.

- Presentación tabular: Esta forma de presentación sirvió para detallar el porcentaje de los resultados aplicados en el método de recolección de datos.

\section{RESULTADOS Y DISCUSIÓN}

\section{Resultados}

A continuación, se describen los resultados mediante tablas que contienen los datos recopilados de las encuestas realizadas a los trabajadores de la empresa investigada, estas se encuentran procesadas de acuerdo a las dimensiones de cada variable:

Variable 1: Manual de procedimientos de créditos y cobranzas.

\section{Tabla $\mathbf{N}^{\circ} 1$.}

Criterios de evaluación de créditos $\left(1^{\circ}\right.$ Dimensión $)$

\begin{tabular}{|c|c|c|c|c|c|c|c|c|c|c|}
\hline \multirow{2}{*}{ INDICADORES } & \multicolumn{2}{|c|}{ Nunca } & \multicolumn{2}{|c|}{$\begin{array}{l}\text { Casi } \\
\text { nunca }\end{array}$} & \multicolumn{2}{|c|}{ A veces } & \multicolumn{2}{|c|}{$\begin{array}{l}\text { Casi } \\
\text { siempre }\end{array}$} & \multicolumn{2}{|c|}{ Siempre } \\
\hline & $\mathbf{N}^{\circ}$ & $\%$ & $\mathbf{N}^{\circ}$ & $\%$ & $\mathbf{N}^{\circ}$ & $\%$ & $\mathbf{N}^{\circ}$ & $\%$ & $\mathbf{N}^{\circ}$ & $\%$ \\
\hline $\begin{array}{l}\text { Se realiza un correcto } \\
\text { procedimiento de evaluación } \\
\text { crediticia a todos los clientes sin } \\
\text { excepción. }\end{array}$ & 0 & $0 \%$ & 0 & $0 \%$ & 3 & $100 \%$ & 0 & $0 \%$ & 0 & $0 \%$ \\
\hline $\begin{array}{l}\text { Se solicita y verifica los } \\
\text { documentos requisitos antes de la } \\
\text { aprobación del crédito. }\end{array}$ & 0 & $0 \%$ & 0 & $0 \%$ & 2 & $66.67 \%$ & 1 & $33.33 \%$ & 0 & $0 \%$ \\
\hline $\begin{array}{l}\text { Se evalúa la capacidad de pago } \\
\text { antes de aprobar un crédito. }\end{array}$ & 0 & $0 \%$ & 0 & $0 \%$ & 2 & $66.67 \%$ & 1 & $33.33 \%$ & 0 & $0 \%$ \\
\hline $\begin{array}{l}\text { Se evalúa el límite de crédito a } \\
\text { otorgar según condición de cada } \\
\text { prestatario. }\end{array}$ & 0 & $0 \%$ & 0 & $0 \%$ & 2 & $66.67 \%$ & 1 & $33.33 \%$ & 0 & $0 \%$ \\
\hline
\end{tabular}

Fuente: Encuesta a los trabajadores de la empresa Darcell Servicios Integrados S.R.L. 
En la tabla $\mathrm{N}^{\circ} 1$ según la primera dimensión "Criterios de evaluación de créditos" se determinó que el $100 \%$ de los trabajadores consideró que a veces se realiza un correcto procedimiento de evaluación crediticia a todos los clientes sin excepción; el $66.67 \%$ consideró que a veces se solicitan y verifican los documentos requisitos antes de la aprobación del crédito; el $66.67 \%$ consideró que a veces se evalúa la capacidad de pago antes de aprobar un crédito; y el $66.67 \%$ consideró que a veces se evalúa el límite de crédito a otorgar según condición de cada prestatario.

\section{Tabla $\mathbf{N}^{\circ} 2$.}

Gestión de cartera de clientes, créditos y cobranzas ( $2^{\circ}$ Dimensión)

\begin{tabular}{|c|c|c|c|c|c|c|c|c|c|c|}
\hline \multirow{2}{*}{ INDICADORES } & \multicolumn{2}{|c|}{ Nunca } & \multicolumn{2}{|c|}{ Casi nunca } & \multicolumn{2}{|c|}{ A veces } & \multicolumn{2}{|c|}{ Casi siempre } & \multicolumn{2}{|c|}{ Siempre } \\
\hline & $\mathbf{N}^{\circ}$ & $\%$ & $\mathbf{N}^{\circ}$ & $\%$ & $\mathbf{N}^{\circ}$ & $\%$ & $\mathbf{N}^{\circ}$ & $\%$ & $\mathbf{N}^{\circ}$ & $\%$ \\
\hline $\begin{array}{l}\text { Se solicita la } \\
\text { información básica y } \\
\text { necesaria a los clientes } \\
\text { y/o prestatarios. }\end{array}$ & 0 & $0 \%$ & 0 & $0 \%$ & 1 & $33.33 \%$ & 2 & $66.67 \%$ & 0 & $0 \%$ \\
\hline $\begin{array}{l}\text { La empresa cuenta con } \\
\text { una cartera de clientes } \\
\text { fieles y con buen } \\
\text { historial de pago. }\end{array}$ & 0 & $0 \%$ & 0 & $0 \%$ & 1 & $33.33 \%$ & 2 & $66.67 \%$ & 0 & $0 \%$ \\
\hline $\begin{array}{l}\text { Se realizan } \\
\text { reprogramaciones de } \\
\text { créditos vencidos. }\end{array}$ & 1 & $33.33 \%$ & 2 & $66.67 \%$ & 0 & $0 \%$ & 0 & $0 \%$ & 0 & $0 \%$ \\
\hline $\begin{array}{l}\text { Se realizan campañas de } \\
\text { exoneraciones de interés. }\end{array}$ & 2 & $66.67 \%$ & 1 & $33.33 \%$ & 0 & $0 \%$ & 0 & $0 \%$ & 0 & $0 \%$ \\
\hline
\end{tabular}

Fuente: Encuesta a los trabajadores de la empresa Darcell Servicios Integrados S.R.L.

De acuerdo a la Tabla $\mathrm{N}^{\circ} 2$ según la segunda dimensión "gestión de cartera de clientes, créditos y cobranzas" se obtuvo que el $66.67 \%$ de los encuestados establece que casi siempre se solicita la información básica y necesaria a los clientes y/o prestatarios; el $66.67 \%$ de los encuestados establece que casi siempre la empresa cuenta con una cartera de clientes fieles y con buen historial de pago; el $66.67 \%$ de los encuestados establece que casi nunca se realizan reprogramaciones de créditos vencidos; y por último, el $66.67 \%$ de los encuestados establece que nunca se realizan campañas de exoneraciones de interés. 


\section{Tabla 3.}

Estrategias de procedimientos de créditos y cobranzas ( $3^{\circ}$ Dimensión)

\begin{tabular}{|c|c|c|c|c|c|c|c|c|c|c|}
\hline \multirow{2}{*}{ INDICADORES } & \multicolumn{2}{|c|}{ Nunca } & \multicolumn{2}{|c|}{$\begin{array}{c}\text { Casi } \\
\text { nunca }\end{array}$} & \multicolumn{2}{|c|}{ A veces } & \multicolumn{2}{|c|}{$\begin{array}{c}\text { Casi } \\
\text { siempre }\end{array}$} & \multicolumn{2}{|c|}{ Siempre } \\
\hline & $\mathbf{N}^{\circ}$ & $\%$ & $\mathbf{N}^{\circ}$ & $\%$ & $\mathbf{N}^{\circ}$ & $\%$ & $\mathbf{N}^{\circ}$ & $\%$ & $\mathbf{N}^{\circ}$ & $\%$ \\
\hline $\begin{array}{l}\text { Se realizan notificaciones de } \\
\text { cobranza a los prestatarios de } \\
\text { créditos vencidos. }\end{array}$ & 3 & $100 \%$ & 0 & $0 \%$ & 0 & $0 \%$ & 0 & $0 \%$ & 0 & $0 \%$ \\
\hline $\begin{array}{l}\text { Cuenta la empresa con un } \\
\text { diagrama de procedimiento de } \\
\text { créditos. }\end{array}$ & 3 & $100 \%$ & 0 & $0 \%$ & 0 & $0 \%$ & 0 & $0 \%$ & 0 & $0 \%$ \\
\hline $\begin{array}{l}\text { Cuenta la empresa con un } \\
\text { diagrama de procedimiento de } \\
\text { cobranzas. }\end{array}$ & 3 & $100 \%$ & 0 & $0 \%$ & 0 & $0 \%$ & 0 & $0 \%$ & 0 & $0 \%$ \\
\hline
\end{tabular}

Fuente: Encuesta a los trabajadores de la empresa Darcell Servicios Integrados S.R.L.

En conformidad con la tabla $\mathrm{N}^{\circ}$ 3, de acuerdo con la tercera dimensión "Estrategias de procedimientos de créditos y cobranzas", se recopiló que el 100\% de los trabajadores considero que nunca se realizan notificaciones de cobranza a los prestatarios de créditos vencidos; el $100 \%$ de los trabajadores considero que nunca la empresa cuenta con un diagrama de procedimiento de créditos; el 100\% de los trabajadores considero que nunca la empresa cuenta con un diagrama de procedimiento de cobranzas.

\section{Variable 2: Morosidad.}

\section{Tabla 4.}

Control de cuentas por cobrar. $\left(1^{\circ}\right.$ Dimensión $)$

\begin{tabular}{|c|c|c|c|c|c|c|c|c|c|c|}
\hline \multirow{2}{*}{ INDICADORES } & \multicolumn{2}{|c|}{ Nunca } & \multicolumn{2}{|c|}{ Casi nunca } & \multicolumn{2}{|c|}{ A veces } & \multicolumn{2}{|c|}{$\begin{array}{c}\text { Casi } \\
\text { siempre }\end{array}$} & \multicolumn{2}{|c|}{ Siempre } \\
\hline & $\mathbf{N}^{\circ}$ & $\%$ & $\mathbf{N}^{\circ}$ & $\%$ & $\mathbf{N}^{\circ}$ & $\%$ & $\mathbf{N}^{\circ}$ & $\%$ & $\mathbf{N}^{\circ}$ & $\%$ \\
\hline $\begin{array}{l}\text { Se identifica y analiza el reporte } \\
\text { de créditos vencidos. }\end{array}$ & 0 & $0 \%$ & 1 & $33.33 \%$ & 2 & $66.67 \%$ & 0 & $0 \%$ & 0 & $0 \%$ \\
\hline $\begin{array}{l}\text { Cuenta la empresa con la } \\
\text { información exacta y verídica } \\
\text { de los clientes morosos. }\end{array}$ & 0 & $0 \%$ & 1 & $33.33 \%$ & 2 & $66.67 \%$ & 0 & $0 \%$ & 0 & $0 \%$ \\
\hline $\begin{array}{l}\text { Cuenta la empresa con un } \\
\text { reporte de los créditos en } \\
\text { proceso de cobranza. }\end{array}$ & 2 & $66.67 \%$ & 1 & $33.33 \%$ & 0 & $0 \%$ & 0 & $0 \%$ & 0 & $0 \%$ \\
\hline
\end{tabular}

Fuente: Encuesta a los trabajadores de la empresa Darcell Servicios Integrados S.R.L.

A partir de la tabla $\mathrm{N}^{\circ} 4$, según la primera dimensión "Control de cuentas por cobrar", se obtuvo que el $66.67 \%$ de los encuestados considera que a veces se identifica y analiza el 
reporte de créditos vencidos; el $66.67 \%$ de los encuestados considera que a veces la empresa cuenta con información exacta y verídica de los clientes morosos; y el $66.67 \%$ considera que nunca cuenta la empresa con un reporte de los créditos en proceso de cobranza.

\section{Discusión}

De acuerdo a la aplicación de la técnica de recolección de datos, y teniendo presente el objetivo principal de la tesis “Analizar si diseñar un manual de procedimientos de créditos y cobranzas va permitir reducir el índice de morosidad en la empresa Darcell Servicios Integrados S.R.L., en la ciudad de Jaén en el periodo 2020”. Desarrollamos la discusión de acuerdo a los resultados obtenidos teniendo como base los objetivos específicos:

O.E.1: "Establecer procesos y criterios de evaluación crediticia en el manual de procedimientos de créditos y cobranzas para reducir la morosidad en la empresa Darcell Servicios Integrados S.R.L

De las encuestas determinamos que existen créditos aprobados por gerencia por poseer vínculos de amistad y no por un correcto procedimiento de evaluación de créditos; adicional a ello se aceptaban la falta de algunos requisitos, no se realizaba una correcta evaluación de acuerdo a la capacidad de pago del prestatario, no se tenía en cuenta el historial crediticio y se ha venido excediendo en los montos de dinero brindado.

Es por ello que teniendo en cuenta la teoría de Strap \& Slao (2018), es de mucha importancia contar con un manual de procedimientos de créditos y cobranzas donde se plasmen los procesos, normatividad, políticas para una correcta evaluación crediticia, pues de esta manera se logrará obtener una cartera de clientes fieles y con buen historial de pago, lo cual permitirá una mejora en el área de crédito y cobranzas y reducirá la morosidad en la empresa.

O.E.2: "Implementar la gestión de cartera de clientes, créditos y cobranzas con relación a la morosidad en la empresa Darcell Servicios Integrados S.R.L".

Este es un punto importante obtenido de la encuesta ya que considerando la importancia del cumplimiento de todos los requisitos que se solicitan, pues se determinó que no se verifican que los datos proporcionados por el cliente, tanto en domicilio (verificar a través de visitas), número de celular, referencias, sean reales o falsos.

Hasta la actualidad se han realizado en un mínimo del total de créditos otorgados reprogramaciones a los clientes con créditos vencidos. Debido a que no cuentan con 
pautas o reglamentos que especifiquen esas facilidades para los clientes morosos; tales como campañas de exoneración de intereses en los créditos vencidos, castigados y judiciales, con la finalidad de que el cliente se interese en el pago de la amortización para beneficio del prestamista para la recuperación del capital.

Una teoría que se relaciona con nuestro objetivo es la de Vega Barrios, K. (2019), el cual considera que contar con un portafolio detallado con los datos importantes y relevantes de los clientes facilita el procedimiento de cobranza, adicional a ello permite clasificar y analizar los tipos de clientes que tiene la empresa, de esa manera facilita para un futuro procedimiento de evaluación crediticia; y por último disminuirá el riesgo de la cartera crediticia.

O.E.3: “Aplicar estrategias en el manual de procedimientos de créditos y cobranzas para disminuir la morosidad de la empresa Darcell Servicios Integrados S.R.L”.

De lo obtenido, la empresa no realiza el procedimiento de cobranzas por no contar con personal adecuado y capacitado para dicha área; adicional a ello no cuenta con diagramas del procedimiento de créditos; adicional a ello no cuenta con un diagrama del procedimiento de cobranzas ya que en la actualidad dicha área no tiene la empresa.

En los últimos periodos pocas veces al finalizar un periodo la empresa se ha preocupado por organizar, realizar y analizar un reporte de los créditos vencidos para visualizar la magnitud o el grado de morosidad que existe en su entidad.

Según los pocos reportes con los que cuenta, podemos notar que algunos datos brindados por los prestatarios no son verídicos o ya no están actualizados. Tampoco existen reportes de créditos en proceso de cobranzas porque la empresa no cuenta con dicha área.

La empresa no se ha interesado por analizar y averiguar las causas de la falta de pagos de cada cliente, de los que tiene conocimiento es de aquellos prestatarios que han informado por voluntad propia el origen del retraso en sus pagos.

Otro resultado obtenido de esta investigación es que la empresa no analiza el avance o deceso de las cuentas por cobrar en comparación a los años anteriores, para ello es de mucha importancia el análisis de sus estados financieros comparativos y sobre todo dar a conocer a los trabajadores para que tengan conocimiento y se preocupen por la realización correcta para la rápida recuperación de la empresa.

Para Tarrillo Díaz, D. (2018), contar con un plan de estrategias de cobranza permite reducir el índice de morosidad con su aplicación, una de las estrategias plasmadas es 
realizar un análisis riguroso de la situación económica financiera de sus clientes, a la vez se debe ofrecer alternativas de solución para los diferentes tipos de morosos, con la finalidad de mejorar la cultura de pago de sus clientes.

Con relación entre la hipótesis y los resultados obtenidos de la aplicación del instrumento se tiene que efectivamente el diseño y aplicación del manual de procedimiento de créditos y cobranzas facilitara el proceso en el otorgamiento de créditos y evaluación el cual permitirá la reducción de morosidad en la empresa Darcell Servicios Integrados SRL.

\section{CONCLUSIÓN O CONSIDERACIONES FINALES.}

\section{Conclusión}

- Se determinó que sólo a veces se realizan los procesos y criterios de evaluación crediticia, concluyendo que existe un déficit en esta etapa principal, de esta forma se evalúa incorrectamente y se brindan créditos elevados sin verificación de información, sin garantía, sin evaluar la capacidad de pago y en su mayoría por aprobación u orden de gerencia generando incremento de morosidad en la empresa a la falta de un manual de procedimientos.

- Se concluyó que la empresa solicita información básica a los clientes, pero no realiza verificación de esta información lo que ocasiona una mala gestión de la cartera de clientes, y en cuanto a la gestión de créditos y cobranzas la empresa durante los últimos años no realizó reprogramaciones ni exoneración de interés de créditos morosos; esto demuestra la incapacidad de parte de la empresa en su gestión para mitigar la morosidad.

- Se concluye que la empresa no cuenta con estrategias para disminuir la morosidad, ya que durante los últimos años nunca se realizó ningún tipo de notificación a la cartera morosa por no contar con el área de cobranzas, esto demuestra la importancia de contar con un manual de procedimientos en esta área.

- Concluimos que la empresa no cuenta con un diagrama de procedimientos de créditos y cobranzas, esto dificulta a los trabajadores realizar correctamente el procedimiento y genera ineficiencia en las actividades dado como resultado incremento de clientes morosos.

\section{Recomendaciones}

- La empresa deberá brindar la Información y conocimiento a cada uno de los trabajadores sobre el manual de procedimientos de créditos y cobranzas, con la 
finalidad de aplicar e implementar el correcto procedimiento en la evaluación y otorgación de un crédito.

- La empresa se encargará de controlar y verificar que la aplicación del manual de procedimientos de créditos y cobranzas se realice correctamente. Es importante que la empresa realice y clasifique periódicamente un reporte de los créditos vencidos y de aquellos que se encuentran en proceso de cobranza con la finalidad de establecer nuevas medidas y continuar con la mejora y actualización de forma y procedimientos de cobranzas.

- La empresa debe de realizar capacitaciones constantes a los trabajadores de esta área, realizar campañas de exoneración de interés de créditos vencidos, identificar las causas de falta de pagos de sus clientes y aplicar estrategias para la recuperación de capital.

- Se recomienda a la empresa realizar análisis de los estados financieros en comparación con años anteriores para determinar la disminución de cartera morosa y la mejora de la utilidad de la empresa.

\section{Propuesta}

\section{MANUAL DE PROCEDIMIENTOS DE CRÉDITOS Y COBRANZAS PRESENTACIÓN}

La empresa "Darcell Servicios Integrados S.R.L.", perteneciente al sector privado constituida el 23 de Julio del año 1999 teniendo como rubro básico el de servicios como son: contables, administrativos, dotación de personal, limpieza, entre otros; y, desde el año 2013 se ha implementado el servicio de créditos para personas naturales trabajadoras de instituciones públicas mediante convenios para dicho fin; a partir del año 2016, se está incorporando a ello el servicio de créditos para micro negocios.

Es por ello, que es necesario diseñar el manual de procedimientos de créditos y cobranzas, con la finalidad de que los colaboradores realicen el correcto proceso, para evitar errores en los diversos procesos del servicio de créditos y cobranzas. Lo cual nos va permitir una adecuada gestión del riesgo crediticio, alineada con las mejores prácticas vigentes, para la determinación de la capacidad de pago de los clientes (deudores), para ello se presenta nuestro organigrama general de la empresa. 
FIGURA $\mathrm{N}^{\circ} 1$ : Organigrama de la empresa.

OBJETIVO

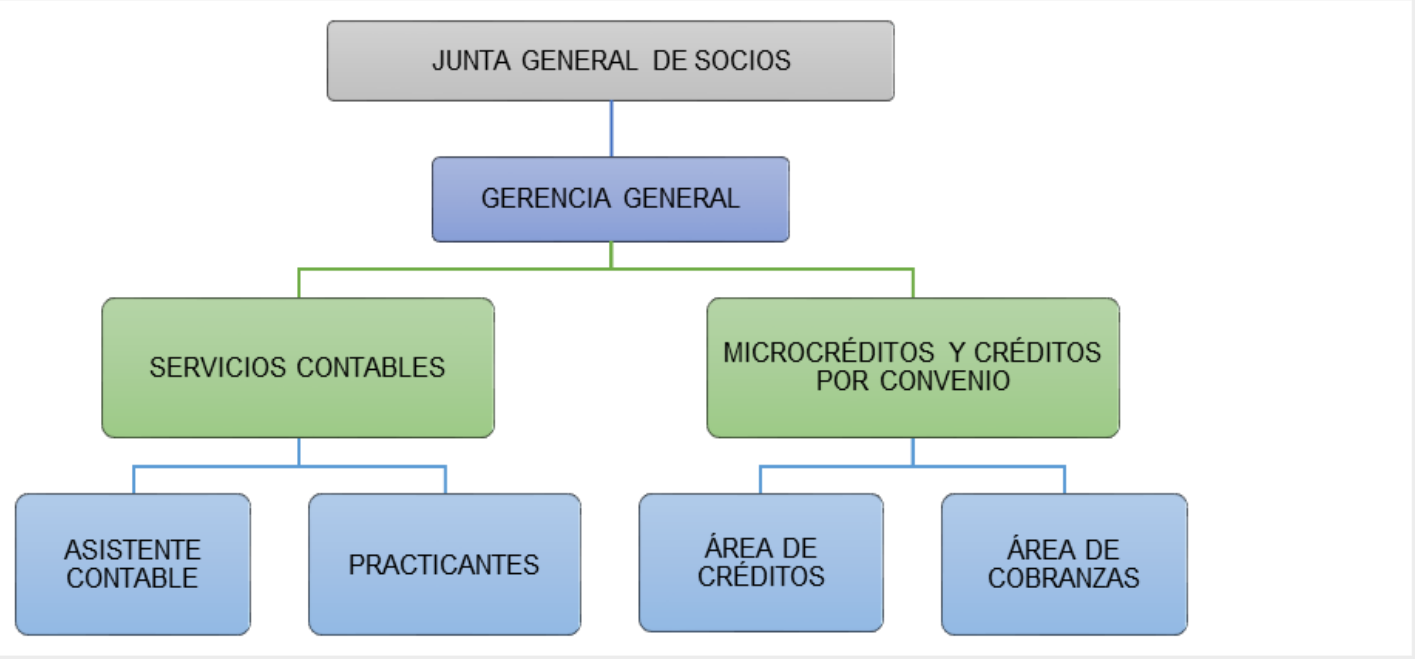

EI objetivo del presente manual de procedimientos de créditos y cobranzas es establecer lineamientos adecuados para la ejecución de las operaciones crediticias en los diversos productos que brinde la empresa, su carácter es estrictamente laboral, ya que este es un instrumento reglamentario que va permitir al personal del área de créditos conocer e implementar aspectos fundamentales como: sus objetivos, funciones y procedimientos plasmados.

\section{ORGANIZACIÓN DE LA RESPONSABILIDAD CREDITICIA ÁREA DE CRÉDITOS}

El área de créditos es un órgano de apoyo, encargado de planificar, organizar, dirigir y ejecutar los procedimientos de evaluación y otorgación de los créditos, siendo de su responsabilidad preparar y adjuntar el expediente con los documentos requisitos para la determinación del nivel de aprobación correspondiente. 
FIGURA N²: Diagrama de procedimiento de créditos.

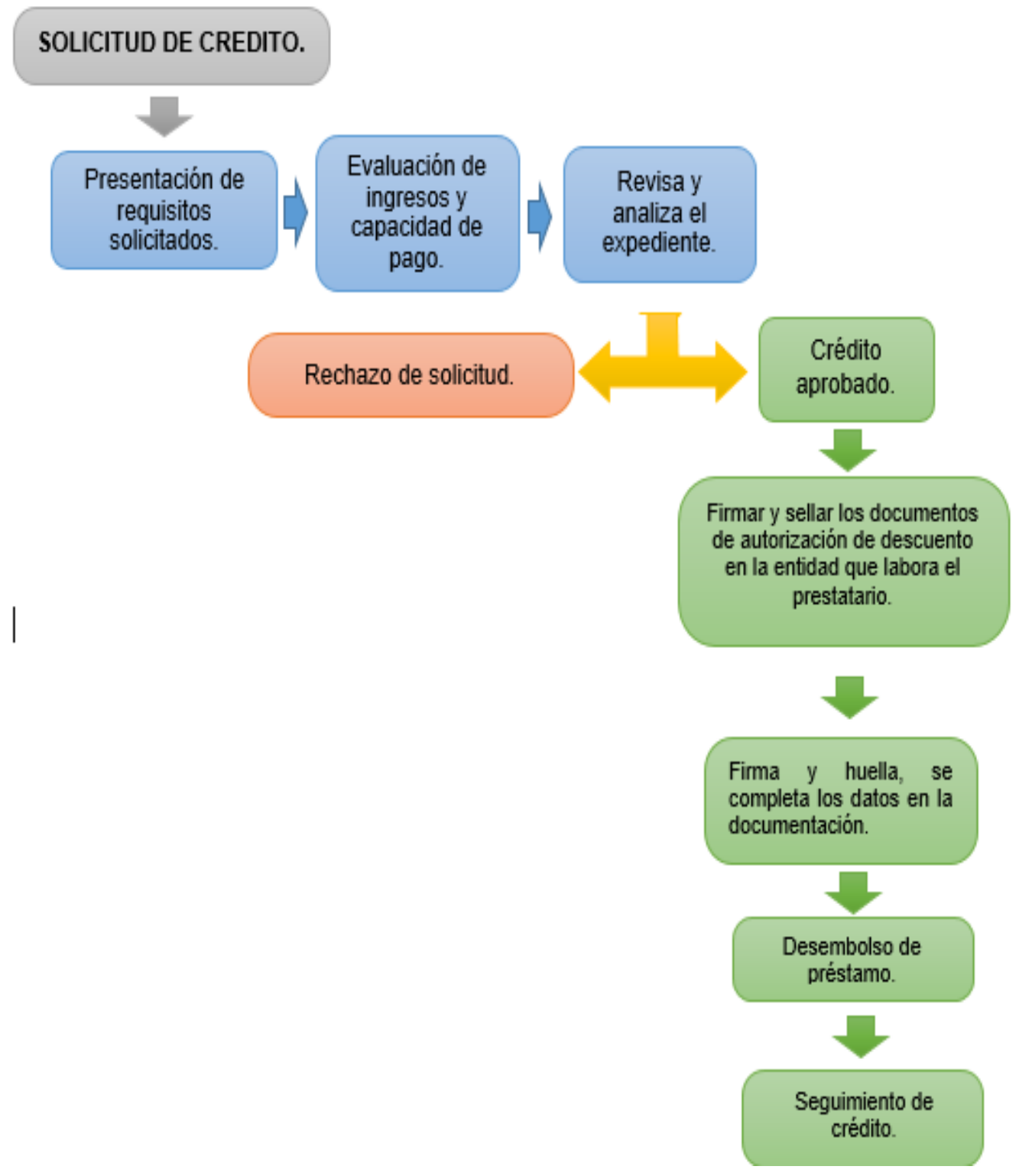

\section{ÁREA DE COBRANZAS}

Está área de cobranza es el órgano encargado de realizar el procedimiento de RECUPERACIÓN DE PRÉSTAMOS desde la cobranza preventiva hasta la cobranza judicial de los créditos otorgados. Cada tipo de cobranza es un proceso distinto. 
FIGURA Nº3: Diagrama de procedimiento de cobranzas.

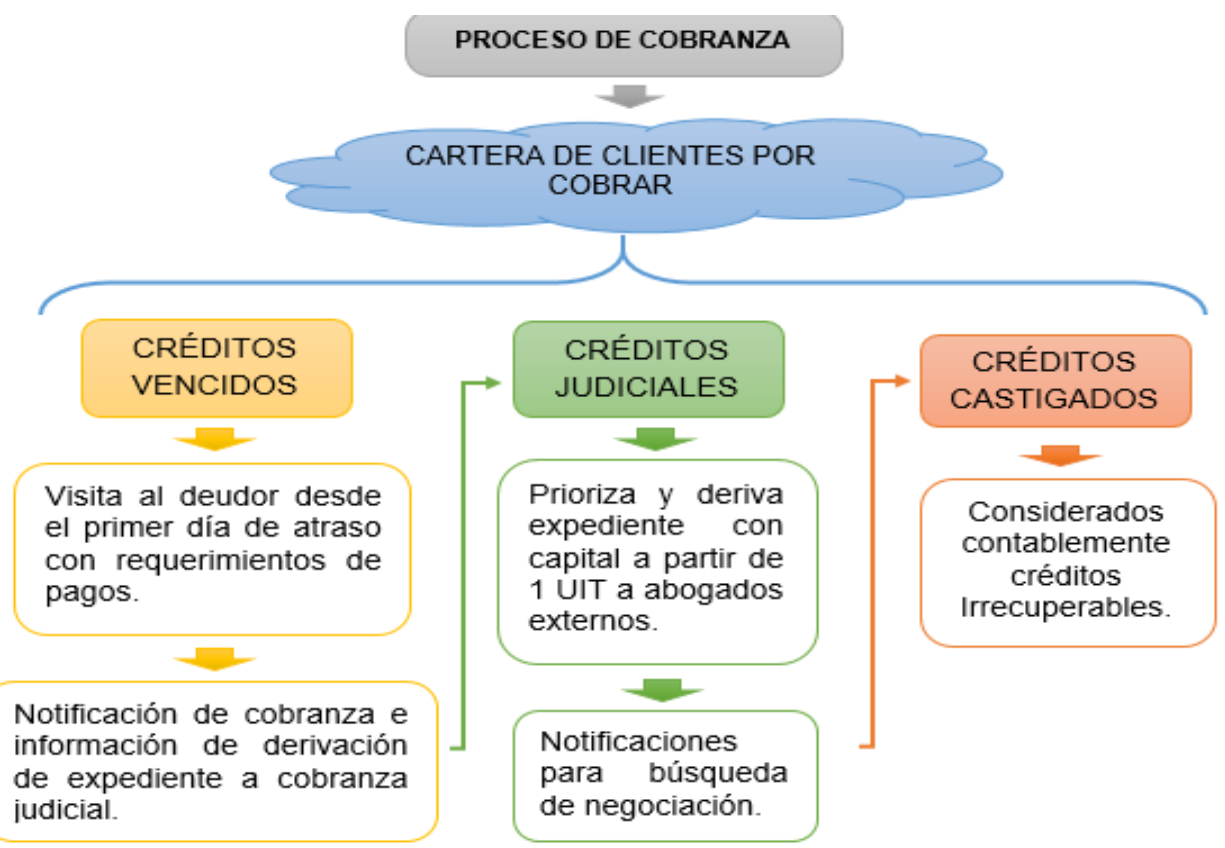

NIVELES DE APROBACIÓN DE CRÉDITOS

\begin{tabular}{|c|l|l|l|l|}
\hline No. & Nivel De Aprobación & \multicolumn{1}{|c|}{$\begin{array}{c}\text { Integrantes Y } \\
\text { Firmas }\end{array}$} & $\begin{array}{c}\text { Límites Crédito } \\
\text { Convenio }\end{array}$ & $\begin{array}{c}\text { Límites Crédito } \\
\text { Micronegocio }\end{array}$ \\
\hline II & Gerencia general. & $\begin{array}{l}\text { Gerente general + } \\
\text { analista de créditos. }\end{array}$ & $\begin{array}{l}\text { Max: 1.50 Min. 10\% } \\
\text { UIT vigente. }\end{array}$ & $\begin{array}{l}\text { Max: 50\% Min. 10\% } \\
\text { UIT vigente. }\end{array}$ \\
\hline I & Analista de créditos. & $\begin{array}{l}\text { Gerente general + } \\
\text { analista de créditos. }\end{array}$ & $\begin{array}{l}\text { Max: 1.00 Min. 10\% } \\
\text { UIT vigente. }\end{array}$ & $\begin{array}{l}\text { Max: 40\% Min. 10\% } \\
\text { UIT vigente. }\end{array}$ \\
\hline
\end{tabular}

\section{LISTA DE REFERENCIAS}

Espín Castro, D. E., \& Campoverde Calderón , A. V. (2018). Diseño del manual de políticas de crédito y cobranzas para Livestravel S.A. Universidad de Guayaquil, Guayaquil.

Acosta Aspajo, D. D. (2015). Plan estratégico para mejorar la fidelización de los clientes de la Cooperativa de Ahorro y Crédito Santo Cristo de Bagazán - Moyobamba 2015. Moyobamba.

Alcívar Moreira , X. K., \& Bravo Santana , V. P. (2020). Índice del riesgo de morosidad en los microcréditos simples; caso Cooperativa de ahorro y crédito Chone LTDA. Revista Científica Multidisciplinaria Arbitrada Yachasun, 4(7), 182-198. Obtenido de https://editorialibkn.com/index.php/Yachasun/article/view/90 
Alvarez Risco, A. (2020). Clasificación de las Investigaciones. Obtenido de Universidad de

Lima:

https://repositorio.ulima.edu.pe/bitstream/handle/20.500.12724/10818/Nota\%20 Acad\%C3\%A9mica\%202\%20\%2818.04.2021\%29\%20-

$\% 20$ Clasificaci\%C3\%B3n\%20de\%20Investigaciones.pdf?sequence=4\&isAllow $\mathrm{ed}=\mathrm{y}$

Bedoya Ospina, J. G. (2017). Credit cycles, global liquidity and monetary regimes: an approach for Latin America. Desarrollo y Sociedad(78), 271-313. Obtenido de https://www.redalyc.org/pdf/1691/169149895008.pdf

Bobadilla, E. (25 de 03 de 2019). "En el 2018 aumentó la morosidad crediticia en el Perú". Gestión.

Céspedes Guillermo, C. V. (2018). La Cartera crediticia y su influencia en el nivel de morosidad de la Cooperativa de Ahorro y Crédito Norandino Ltda, de la Ciudad de Jaén 2016. Jaén.

Chavarín Rodríguez, R. (2015). Default in the payment of credits and profitability of commercial banks in Mexico. Mexican journal of economics and finance, 10(1).

Chavarín Rodríguez, R. (2015). Delay in the payment of credits and profitability of commercialbanking in Mexico. Revista Mexicana de Economía y Finanzas., 10(1), 71-83.

Chavez Moreno, E. G. (2017). Proposal to improve the credit and collections process to optimize liquidity in the company Hellmann Worldwide Logistics S.A.C. Lime.

Cobián Salazar, K. S. (2015). Gestión de créditos y cobranzas y su efecto en la rentabilidad de la Mype San Pedro EIRL. Trujillo, 2015. TRUJILLO.

Contreras Gómez, L. (2020). Delinquency of the consumer loan portfolio and its impact on the profitability and liquidity of Banco Mercantil, Banco Universal. Gestión y desarrollo Libre, 5, 9.

Coronel Huamán, L. (2016). Estrategias de cobranza y su relación con la morosidad en la entidad Financiera Mi Banco- Jaén 2015. Jaén.

Espinoza Sánchez, S., Aquino Condor, W., Lima Huamaní, O., Villafuerte Alcántara, R., Sánchez Garcés, J., \& Mamani Monrroy, K. (2020). La morosidad: factores motivacionales de 1 os responsables financieros, en el contexto del COVID - 19. Valor Contable, 7(1), 37-49. 
Gallardo Echenique, E. E. (2017). Metodología de la investigación. Manual autoformativo interactivo. Universidad Continental, 21. Obtenido de https://repositorio.continental.edu.pe/bitstream/20.500.12394/4278/1/DO_UC_E G_MAI_UC0584_2018.pdf

García Zambrano, X., Maldonado Pazmiño, H., Galarza Morales, C., \& Grijalva Guerrero, G. (2019). Control interno a la gestión de créditos y cobranzas en empresas comerciales minoristas en el Ecuador. Ecuador.

Garrido, J. M. (2018). Ega Futura Nube. Obtenido de Ega Futura Nube: https://www.egafutura.com/glosario/morosidad

Gonzales Pisco, E., \& Sanabria Bolaños, S. (2016). Gestión de cuentas por cobrar y sus efectos en la liquidez en la facultad de una universidad particular, Lima, periodo 2010-2015. Investigación Universitaria., 5(2).

Hinostroza Hermoza, H. (2021). Gestión crediticia y la morosidad del Banco Pichincha del Perú, periodo 2019. Quipukamayoc, 29(59), 69-75.

Krichene, A. (2017). Using a naive Bayesian classifier methodology for loan risk assessment: Evidence from a Tunisian commercial bank. Journal of Economics, Finance and Administrative Science, 22(42), 3-24. Obtenido de http://www.scielo.org.pe/scielo.php?pid=S2077$18862017000100002 \&$ script=sci_abstra

Lozano, G., \& A, L. I. (2017). Diseño manual politicas de créditos y cobranzas para mejorar liquidez de la empresa Química S.A. Guayaquil.

Mata Solís, L. D. (30 de Julio de 2019). Diseños de investigaciones con enfoque cuantitativo de tipo no experimental. Obtenido de Investigalia: https://investigaliacr.com/investigacion/disenos-de-investigaciones-conenfoque-cuantitativo-de-tipo-no-experimental/

Mogollón Gómez, J. (2021). Gestión de cobranza y su impacto en la gerencia financiera de la empresa PP S.A: Periodo 2014-2016. Investigación Científica, 3(1), 262285.

Moreno, J. (24 de Enero de 2018). Evolution of bank delinquency in Spain and Europe. Asesores Financieros.

Murillo Gili, R. (2019). The credit cycle in America: How much should we be Concerned About? Part III. Mercados financieros-Focus, 9-10. 
Paredes Paredes, E. (2019). “Calificación crediticia y otorgamiento de créditos en la Banca Comercial de Lima Metropolitana, 2011 - 2018”. Universidad Nacional Federico Villareal, Lima, Lima.

Pedrosa, S. J. (2016). Delinquency. Economipedia.com. Obtenido de https://economipedia.com/definiciones/morosidad.html

Pérez Coronell, L., López Polo , L., \& De la Hoz Granadillo, E. (2017). Modelo de gestión de relaciones con los clientes en empresas de consultoria. Investigación e Innovación de Ingenierias, 5(2), 46-77.

Periche Delgado, G., Ramos Farroñan, E., \& Chamolí Falcón, A. (2020). La morosidad ante un confinamiento del Covid-19 en la Caja Rural de Ahorro y Crédito Raíz, Perú. Investigación Valdizana. Obtenido de http://revistas.unheval.edu.pe/index.php/riv/article/view/801/732

Ramos Galarza, C. (2020). Los Alcances de una investigación. Ciencia Ámerica, 9(3). Obtenido de http://dx.doi.org/10.33210/ca.v9i3.336

Roldán, P. N. (07 de junio de 2017). Bank delinquency. Economipedia.com. Obtenido de https://economipedia.com/definiciones/morosidad-bancaria.html

Solano, B., \& Gómez, G. (2016). Proposal for a credit and collection manual to improve liquidity. Guayaquil.

Solano, K. G. (2020). Manual design of internal control procedures for the accounting department of sports federations in the Ecuadorian highlands, case study. $\begin{array}{llll}\text { Espacios, } & \text { 41(14), } & \text { Obtenido }\end{array}$ http://www.revistaespacios.com/a20v41n14/20411413.html

Strap Mendoza, P. G., \& Slao Morante, K. I. (Febrero de 2018). Preparation of a procedure manual for the department of credit and collections in the Company Otelo \& Fabell. Revista Observatorio de la Economía Latinoamericana. Obtenido de //www.eumed.net/2/rev/oel/2018/02/manual-otelo-fabell.html

Suárez Hurtado, V. M. (2018). “Diseño de un manual de políticas de créditos y cobranzas para disminuir la cartera vencida de la empresa Mueblerías Palito S.A., periodo 2017-2018”. Guayaquil.

Tarrillo Diaz, D. O. (2018). Plan Estratégico de cobranzas para reducir el índice de morosidad en la Ong Adra Perú en la Agencia Jaén-2017. Jaén. 
Tenorio Almache, J. L., Tovar Arcos, G. R., \& Almeida Vásquez, O. I. (2019). Los manuales de procedimientos como base sólida de un emprendimiento. Científica Ciencias Económicas y Empresariales, 4(1), 194-210.

Vásquez Rodríguez, J. A. (Diciembre de 2016). El manual maestro de procesos y procedimientos, un paso hacia la gestión de calidad: el caso del Consejo Nacional de Vialidad. Nacional de Administración, 7(2), 121-141. Obtenido de https://revistas.uned.ac.cr/index.php/rna/article/view/1516/1968

Vega Barrios, K. J. (2019). Company portfolio procedure manual Colpromed Sas. Universidad Cooperativa de Colombia, Bucaramanga. Obtenido de https://repository.ucc.edu.co/bitstream/20.500.12494/12874/4/2019_Manual\%20 procedimiento.pdf

Vivanco Vergara, M. E. (2017). Los manuales de procedimientos como herramientas de control interno de una organización. Universidad y Sociedad, 9(3), 2218-3620. 